\title{
Imaging findings of hepatic focal nodular hyperplasia in men and women: are they really different?
}

\author{
Tommaso Vincenzo Bartolotta • Adele Taibbi • \\ Giuseppe Brancatelli $\cdot$ Domenica Matranga • \\ Marisa Tumbarello $\cdot$ Massimo Midiri $\cdot$ Roberto Lagalla
}

Received: 10 January 2013/Accepted: 13 May 2013/Published online: 3 December 2013

(C) Italian Society of Medical Radiology 2013

\begin{abstract}
Purpose This study was undertaken to compare the imaging findings of focal nodular hyperplasia (FNH) in men and women, as seen on multidetector computed tomography (MDCT), magnetic resonance imaging (MRI) and contrast-enhanced ultrasound (CEUS).

Materials and methods Two radiologists reviewed 195 imaging studies (17 MDCT, 81 MRI and 97 CEUS examinations) pertaining to $111 \mathrm{FNHs}$ (mean size $3 \mathrm{~cm}$ ) in 91 patients (mean age 39 years). For each lesion, the readers assessed size, location, echogenicity, attenuation, or signal intensity in comparison with adjacent liver parenchyma on both unenhanced and postcontrast images. Results Eighty-nine FNHs (mean size $3.1 \mathrm{~cm}$ ) were observed in 73 women (mean age 37.9 years) and 22 FNHs (mean size $2.7 \mathrm{~cm}$ ) in 18 men (mean age 41.2 years). No statistically significant differences were found between men and women in terms of age, FNH lesions per patient (1.22 and 1.21, respectively), size, baseline and enhancement pattern on MRI, CEUS and MDCT $(p<0.05)$. A central scar in FNHs was depicted in 4/18 (22.2\%) men and $16 / 63(25.4 \%)$ women on MRI $(p<0.05)$, and in $1 / 2$ $(50 \%)$ men and $7 / 15(46.7 \%)$ women on MDCT $(p<0.05)$, whereas a spoke-wheel pattern, central scar, and/or feeding vessel were seen in 5/17 $(29.4 \%)$ men and 22/80 (27.5\%) women on CEUS $(p<0.05)$.
\end{abstract}

T. V. Bartolotta ( () ) A. Taibbi · G. Brancatelli ·

D. Matranga $\cdot$ M. Tumbarello $\cdot$ M. Midiri · R. Lagalla

Department of Radiology, University of Palermo,

Via Del Vespro 129, 90127 Palermo, Italy

e-mail: tv_bartolotta@yahoo.com;

tommasovincenzo.bartolotta@unipa.it
Conclusions Our results did not show any differences in imaging features, age of occurrence and size of FNH between men and women.

Keywords Focal nodular hyperplasia - Liver . Computed tomography $\cdot$ Magnetic resonance imaging . Contrast-enhanced ultrasound

\section{Introduction}

Hepatic focal nodular hyperplasia $(\mathrm{FNH})$ is the second most common liver tumour, the incidence of which is rising as a result of the widespread use of cross-sectional imaging. FNH may occur in both sexes and at all ages, but it is found most commonly in women (80-95\% of cases) in their third to fourth decades of life, with a female-tomale ratio of up to $5: 1$ [1].

Although the imaging findings of FNH are well established, there is some controversy about the characteristics of FNH with regard to patient gender [1, 2]. Luciani et al. [3] reported that in men the mean age at diagnosis was significantly higher, the size was significantly smaller and FNH showed more often atypical imaging findings than those in women. Conversely, Nguyen et al. [4] reported that the distribution of the various morphological forms between women and men was not significantly different. Furthermore, several studies have demonstrated that detection of the typical imaging features of FNH is often heavily dependent on lesion size, being less frequent in lesions smaller than $3 \mathrm{~cm}$ [5-7]. We, therefore, undertook this study in an attempt to clarify whether radiologists should expect any gender differences in the imaging findings of $\mathrm{FNH}$ detected in men or women. 


\section{Materials and methods}

Patient population and imaging techniques

Institutional review board approval was obtained and full informed consent was waived for this retrospective study. Our study complied with the terms of the Declaration of Helsinki [8].

We searched our hospital's medical records (radiology, pathology, surgical pathology and discharge summary) to identify patients with FNH treated during a 6-year period (from January 2006 to December 2011). Patients were eligible for enrolment on the basis of the following inclusion criteria: (1) a conclusive diagnosis of FNH (see reference standard); (2) they had undergone at least one of the following imaging studies: (a) baseline and contrastenhanced ultrasound (CEUS) scan; (b) multiphase multidetector computed tomography (MDCT) scan; (c) magnetic resonance imaging (MRI) with hepatocellular-specific contrast agent. Patients were excluded from the analysis if the imaging protocol was suboptimal or if the images could not be retrieved from our imaging archives.

\section{Computed tomography}

CT studies were performed with a 64-slice MDCT scanner (Brilliance 64, Philips Medical Systems, Eindhoven, The Netherlands). Patients received $1.5 \mathrm{~mL} / \mathrm{kg}$ total body weight of $400 \mathrm{mgI} / \mathrm{mL}$ nonionic contrast agent (Iomeron 400, Bracco Imaging, Milan, Italy) infused with an automated injector (Medrad, Indianola, IA, USA) at a rate of $4 \mathrm{~mL} / \mathrm{s}$ through a 18-20 gauge catheter inserted into an antecubital vein. CT was performed immediately before contrast agent administration and during the hepatic arterial, hepatic venous and delayed phases. To determine the scanning delay for the hepatic arterial phase, the time-topeak aortic enhancement was assessed using an automatic bolus-tracking technique with automated scan-triggering software (Bolus Pro Ultra, Philips Medical Systems, Eindhoven, The Netherlands). Hepatic arterial phase scanning was started automatically $18 \mathrm{~s}$ after the trigger threshold $(150 \mathrm{HU})$ reached the level of the suprarenal abdominal aorta. The hepatic venous and delayed phases were acquired $40 \mathrm{~s}$ after the beginning of the arterial phase and $180 \mathrm{~s}$ after injection of contrast, respectively.

Magnetic resonance imaging

MRI was performed with a $1.5 \mathrm{~T}$ MR unit (Signa Excite HDXT, General Electric Healthcare, Milwaukee, WI, USA) using a phased-array multicoil. The MRI protocol included precontrast axial breath-hold and respiratorytriggered T2-weighted fast spin-echo (FSE) sequences both with and without fat saturation (TR/TE 4,000/76 ms, flip angle $150^{\circ}$, section thickness $6 \mathrm{~mm}$ ), unenhanced (in-phase and out-of-phase) T1-weighted sequences (TR/TE $150 / 4.2-2.1 \mathrm{~ms}$, flip angle $80^{\circ}$, section thickness $4 \mathrm{~mm}$ ) and precontrast fat-saturated T1-weighted spoiled 3D gradient-recalled echo (GRE) sequences (TR/TE $4.2 / 2.0 \mathrm{~ms}$, flip angle $12^{\circ}$, section thickness $3 \mathrm{~mm}$ ). A triphasic dynamic contrast-enhanced study was obtained after the administration of an IV bolus of $0.1 \mathrm{mmol} / \mathrm{kg}$ of gadobenate dimeglumine (MultiHance, Bracco, Italy) into an antecubital vein at a flow rate of $2 \mathrm{~mL} / \mathrm{s}$ through a 20-gauge intravenous catheter by means of a power injector (MR Spectris; Medrad, Pittsburgh, PA, USA) and flushed by $20 \mathrm{~mL}$ of sterile saline solution. Images were acquired using an automated bolus-detection technique (Smartprep technique, GE Healthcare) during the arterial (14 s after bolus injection), hepatic venous and delayed phase (60 and $180 \mathrm{~s}$ after bolus injection, respectively). The dynamic study was followed by a hepatocellular-specific phase obtained $2 \mathrm{~h}$ after the injection of contrast material, with the same scanning parameters.

\section{Contrast-enhanced ultrasound}

Two experienced radiologists (more than 5 years of CEUS of the liver), who were aware of the patients' clinical histories, performed US scanning using either an HDI 5000 (ATL, Bothell, Wash, USA) or an iU22 unit (Philips Ultrasound, Bothell, Wash, USA), both of them equipped with C5-2/C5-1 convex-array probes and pulse inversion imaging software. A baseline survey, including colour/ power Doppler (CD/PD) and spectral analysis, was performed. Once the US scan parameters had been set-such as focal zone and time gain compensation-they were not changed throughout the study. The US contrast agent used in the present study was SonoVue (Bracco, Milan, Italy), which was injected intravenously as a $2.4 \mathrm{~mL}$ bolus followed by $10 \mathrm{~mL}$ of normal sterile saline flush using a 20 or 22-gauge peripheral intravenous cannula. A low frame rate $(5 \mathrm{~Hz})$ and a very low mechanical index (MI), ranging from 0.05 to 0.08 , were used for real-time imaging. One focus was positioned below the level of the lesion. Each examination lasted about $5 \mathrm{~min}$ after bolus injection. No adverse events were recorded either during or immediately after the injection of contrast agent. In patients with multiple lesions, a $2.4 \mathrm{~mL}$ further bolus of SonoVue was administered for each lesion, with an interval time at least of $15 \mathrm{~min}$ to allow for clearance of the previously injected contrast.

Digital cineloops were recorded during both baseline and postcontrast US in the arterial, hepatic venous and extended hepatic venous or late phase (5-40 s, 55-90 s and up to $200-300 \mathrm{~s}$ from the beginning of injection, 
respectively). All images and cineloops were digitally stored as raw data in a PC-based workstation connected to the US units via a standard Ethernet link and sent to our PACS (Impax, Agfa-Gevaert, Milan, Italy).

Image analysis

Two abdominal radiologists (more than 10 years of experience) randomly reviewed all imaging studies by consensus. Neither of the readers was involved in the scanning and both were blinded to the final diagnosis, as well as to the identity, clinical histories and other imaging findings of the patients. Five consecutive interpretation sessions, with a seven-day interval to prevent recall bias, were held to complete the review process.

On each imaging modality, the two readers were asked to report on the size and segment location of each lesion according to the Couinaud classification system and to visually assess echogenicity/attenuation/signal intensity (the latter in all MRI sequences) in comparison with adjacent liver parenchyma on both the unenhanced and the contrast-enhanced images obtained during the arterial, hepatic venous and delayed (or extended hepatic venous for CEUS) phases. For MRI studies, the hepatocellularspecific phase was also assessed. For US and CEUS studies, the colour Doppler images and spectral waveforms were also evaluated for each lesion, as were the following parameters [9-11]:

- "central scar": a central or eccentric hypo- or hyperechoic area at baseline US and/or unhenancing at CEUS in the arterial and hepatic venous and extended hepatic venous phase, also showing distinctly different attenuation/intensity on unenhanced scans or at different phases of enhancement.

- "feeding vessel": an arterial vessel, appreciable at baseline $\mathrm{CD} / \mathrm{PD}$ and/or at CEUS in the arterial phase, branching from the hepatic arterial tree and directed towards the lesion and penetrating it;

- "spoke-wheel" sign: a radial arterial vascularity within the lesion appreciable at baseline colour and power Doppler and/or centrifugal enhancement of the lesion with a central vessel branching from the centre towards the periphery at CEUS in the arterial phase.

- presence and type (arterial or venous) of any intralesional flow, other than the above signs, at baseline colour and power Doppler examination.

\section{Reference standard}

The final diagnosis was established by core biopsy performed with an $18-\mathrm{G}$ needle $(n=1)$ or by demonstrating that FNH was iso- or hyperintense to the surrounding liver in the hepatobiliary phase of MRI $(n=81)$. For the remaining 29 lesions, a combination of size stability during a follow-up period of at least 1 year and imaging features consistent with FNH was used.

The CEUS diagnostic criteria for FNH were based on arterial phase centrifugal filling and stellate vascularity, followed by sustained contrast enhancement on the hepatic venous phase and extended hepatic venous or late phase [10]. The CT diagnostic criteria were: mild hypoattenuation or isoattenuation on precontrast scan; rapid, homogeneous and strong enhancement in the arterial phase except for the central scar; near isoattenuation to liver parenchyma in the hepatic venous and delayed phases. The MRI diagnostic criteria were: iso- or slight hypointensity on T1weighted images; iso- or slight hyperintensity on T2weighted images; hyperintense central scar on T2-weighted images; marked and homogeneous enhancement in the arterial phase except for the central scar; iso- or slight hyperintensity to liver parenchyma in the hepatic venous and delayed phases $[6,7,12]$.

Statistical analysis

Statistical analysis was performed by a biostatistician involved in the study design, using a computer software package (Intercooled Stata for Windows, v. 9.2., StateCorp, TX, USA). To assess the statistical significance of the difference between two genders with respect to US, colour Doppler, CEUS, MDCT and MRI patterns and with respect to the presence and appearance of the central scar, the $\mathrm{z}$ test for proportions or the Fischer exact test were used, as appropriate. Statistical significance was considered to be present at a $p$ value of $<0.05$.

\section{Results}

Overall, 195 complete imaging studies pertaining to 111 FNHs (size range $0.5-9.2 \mathrm{~cm}$, mean $3 \pm 1.8 \mathrm{~cm}$ ) in 91 patients were retrieved (Table 1). There were 73 women and 18 men (age range 18-77 years, mean $39 \pm 14$ years).

Table 1 Number and type of assessed imaging studies

\begin{tabular}{lclllr}
\hline & No. of lesions & MDCT & MRI & CEUS & Total \\
\hline Men & 22 & 2 & 18 & 17 & 37 \\
Women & 89 & 15 & 63 & 80 & 158 \\
Total & 111 & 17 & 81 & 97 & 195 \\
$p$ value & & 0.365 & 0.297 & 0.111 & \\
\hline
\end{tabular}

MDCT multidetector computed tomography, MRI magnetic resonance imaging, CEUS contrast-enhanced ultrasound 
Fig. 1 Focal nodular

hyperplasia (FNH) in a 48-year-

old man, multidetector

computed tomography

(MDCT). a Unenhanced scan

does not show any focal liver

lesion. b During the arterial phase a markedly hypervascular lesion (arrow) is evident in segment VIII showing a tiny hypoattenuating central area corresponding to the central scar (arrowhead). c, d The lesion appears isoattenuating with respect to the surrounding liver parenchyma during the portal venous and delayed phases
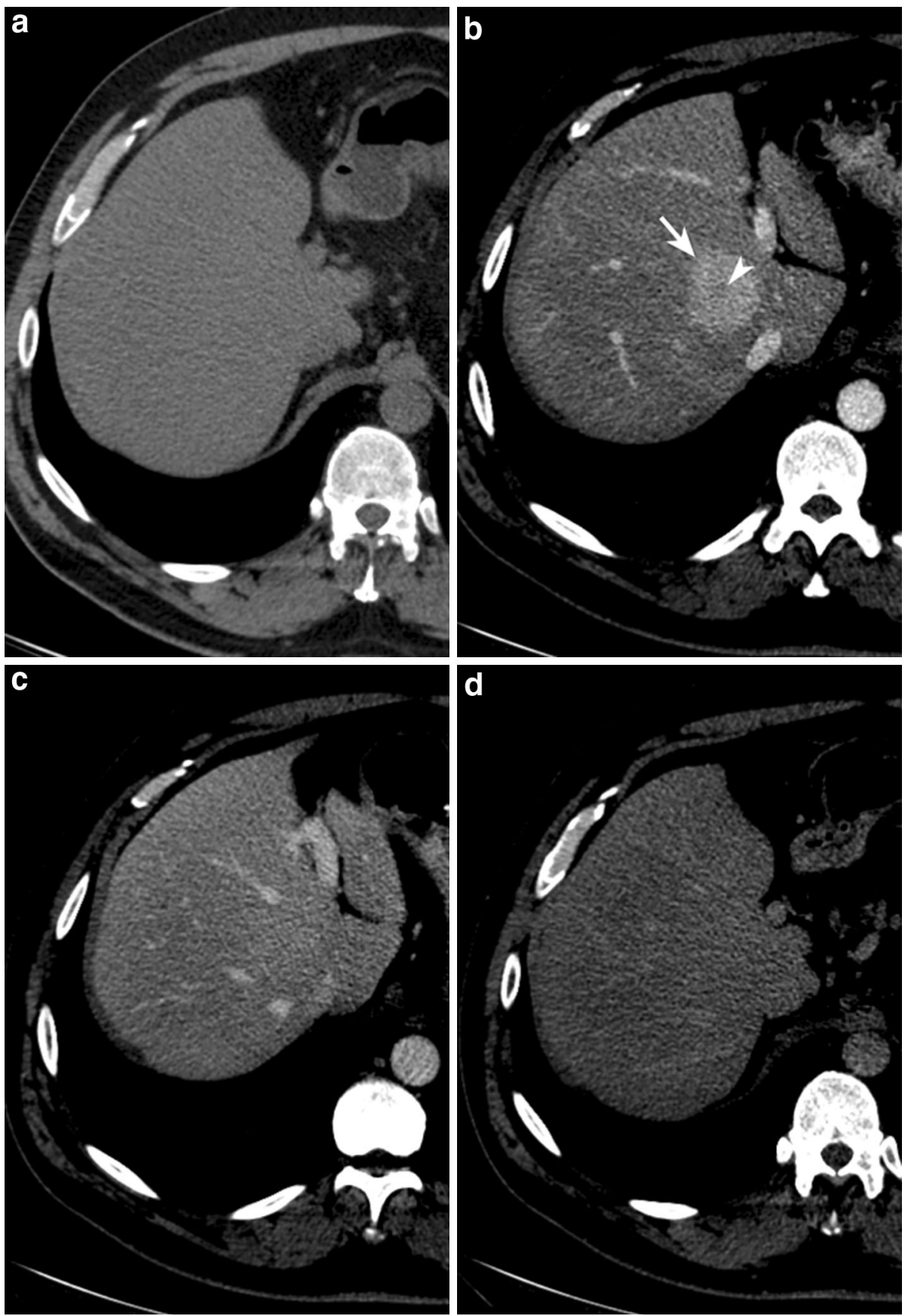

Eighty-nine FNHs (size range $0.5-7.6 \mathrm{~cm}$, mean $3.1 \pm 1.7 \mathrm{~cm}$ ) were observed in 73 women (age range 18-77 years, mean $37.9 \pm 14.4$ years) and 22 FNHs (size range $0.7-9.2 \mathrm{~cm}$, mean $2.7 \pm 1.9 \mathrm{~cm}$ ) in 18 men (age range 25-76 years, mean $41.2 \pm 16.9$ years). Men accounted for $19.8 \%$ of all patients. No statistically significant differences were found between the two groups in terms of lesion size $(p>0.05)$ or mean number of FNH lesions per patient (1.22 vs. 1.21 FNH per patient in men and women, respectively) $(p>0.05)$.
Seventy-six patients $(83.5 \%)$ had one lesion, whereas in the 15 patients who had more than one lesion (11 patients with two lesions, three patients with three lesions and one patient with four lesions), each lesion was studied separately. Seventy-three $(65.8 \%)$ lesions were located in the right liver and the remaining $38(33.2 \%)$ in the left liver.

\section{Computed tomography}

Eleven out of the $17(64.7 \%)$ FNHs studied by means of MDCT were slightly hypoattenuating, whereas $4 / 17$ 
Table 2 Multidetector CT pattern of 17 focal nodular hyperplasia (FNH) lesions

\begin{tabular}{|c|c|c|c|c|c|c|}
\hline & No. of lesions & Unenhanced & Arterial & Hepatic venous & Delayed & Scar \\
\hline \multirow[t]{2}{*}{ Men } & $2 / 17(11.8 \%)$ & 1 Iso & 2 Hyper & 2 Hyper & 1 Iso & 1 Нуро \\
\hline & & 1 Нуро & & & 1 Hyper & \\
\hline \multirow[t]{3}{*}{ Women } & $15 / 17(88.2 \%)$ & 10 Нуро & 15 Hyper & 13 Iso & 15 Iso & 7 Нуро \\
\hline & & 3 Iso & & 2 Hyper & & \\
\hline & & 2 Hyper & & & & \\
\hline$p$ value & & 0.596 & 1.000 & 0.044 & 0.118 & 1.000 \\
\hline
\end{tabular}

Table 3 Contrast-enhanced magnetic resonance imaging (MRI) patterns in $81 \mathrm{FNHs}$

\begin{tabular}{lllll}
\hline Men & Women & Arterial & Portal venous & Delayed \\
\hline $16(88.9 \%)$ & $52(82.5 \%)$ & Hyperintense & Hyperintense & Hyperintense \\
$2(11.1 \%)$ & $10(15.8 \%)$ & Hyperintense & Isointense & Isointense \\
- & $1(1.5 \%)$ & Hyperintense & Hypointense & Isointense \\
18 & 63 & $p$ value $=0.786$ & & \\
\hline
\end{tabular}

$(23.5 \%)$ were isoattenuating and 2/17 (11.8\%) were slightly hyperattenuating on the baseline unenhanced images $(p=0.013)$.

After contrast agent injection, 17/17 (100\%) FNHs became hyperattenuated to varying degrees in comparison with adjacent liver parenchyma in the arterial phase and 13 of them $(76.5 \%)$ appeared isoattenuating both in the hepatic venous and delayed phases, whereas 4/17 (23.5\%) FNHs appeared hyperattenuating in the hepatic venous phase. In the delayed phase, these four lesions were either isoattenuating $(n=3)$ or hyperattenuating $(n=1)$.

A central scar was depicted on MDCT images as an unenhancing hypoattenuating central area in 7/15 (46.7 \%) FNHs occuring in women and in one of the two (50\%) FNHs in men. No statistically significant differences were found between men and women (Fig. 1; Table 2).

Magnetic resonance imaging

Sixty-two out of $81(76.5 \%)$ FNHs studied with MRI were slightly hypointense on baseline T1-weighted images and $72 / 81(88.9 \%)$ were slightly hyperintense on baseline T2weighted images $(p=0.038)$. No statistically significant difference between men and women was found: 14/18 (77.8 \%) vs. 48/63 (76.2\%) FNHs were hypointense on T1-weighted images and 16/18 (88.9\%) vs. 56/63 $(88.9 \%)$ FNHs were hyperintense on T2-weighted images in men and women, respectively $(p>0.05)$. The remaining four FNHs in men were isointense both on T1-weighted (4/ $18 ; 22.2 \%)$ and $\mathrm{T} 2$-weighted $(2 / 18 ; 11.1 \%)$ images, whereas the remaining $15 \mathrm{FNHs}$ in women were isointense on T1-weighted images $(15 / 63 ; 23.8 \%)$, and showed isointensity $(6 / 63 ; 9.5 \%)$ or hypointensity $(1 / 63 ; 1.5 \%)$ on
T2-weighted images, without any statistically significant difference between men and women $(p>0.05)$.

After contrast agent injection, 81/81 (100\%) FNHs became hyperintense to varying degrees in comparison with adjacent liver parenchyma in the arterial phase and 68 of them $(83.9 \%)$ appeared hyperintense in both the hepatic venous and delayed phases $(p=0.0002)$, whereas $12 / 81$ $(14.8 \%)$ FNHs showed isointensity in the hepatic venous and delayed phases (Table 3; Fig. 2). One FNH showed slight hypointensity in the hepatic venous phase but appeared isointense in the delayed phase. All $81 \mathrm{FNHs}$ showed uptake of Gd-BOPTA in the hepatocellular-specific phase, appearing either hyperintense $(70 / 81 ; 86.4 \%)$ or isointense (11/81; $13.6 \%$ ) (Fig. 2). No statistically significant differences were found between men and women (Table 3).

A central scar could be detected on MRI in 20/81 (24.7\%) FNHs, without any statistically significant difference between men $(4 / 18 ; 22.2 \%)$ and women $(16 / 63$; $25.4 \%$ ) (Fig. 2; Table 4). The central scar appeared more frequently hypointense in men than in women in the hepatic venous phase $(p<0.006)$ (Table 4$)$.

\section{US, colour Doppler US and CEUS}

Seventy-two out of the $97(74.2 \%)$ FNHs studied by means of US were hypoechoic on baseline grey-scale US images, but no statistically significant difference between men and women was found $(p>0.05)$.

At colour Doppler US at least one sign-spoke-wheel, central scar, and/or feeding vessel sign_-could be detected in 37/97 (38.1\%) FNHs, without any statistically significant difference between females and males $(p>0.05)$. 
Fig. 2 FNH in a 31-year-old man, magnetic resonance imaging (MRI). a Unenhanced MR shows a $3.5-\mathrm{cm}$ isointense (arrow) lesion in segment VI which shows a small central scar that is hypointense with respect to the surrounding liver parenchyma on T1-weighted imaging (arrowhead). b The lesion is slightly hyperintense (arrow) but with a more hyperintense central scar on the T2-weighted image

(arrowhead). c In the arterial phase the lesion shows marked and homogeneous contrast enhancement (arrow) except for the hypointense central scar (arrowhead). d The lesion appears hyperintense during the portal venous and delayed phases (e) (arrows), whereas the central scar appears constantly hypointense (arrowheads). f Two hours after the injection of Gd-BOPTA the lesion shows contrast uptake, appearing slightly hyperintense in comparison to the adjacent liver
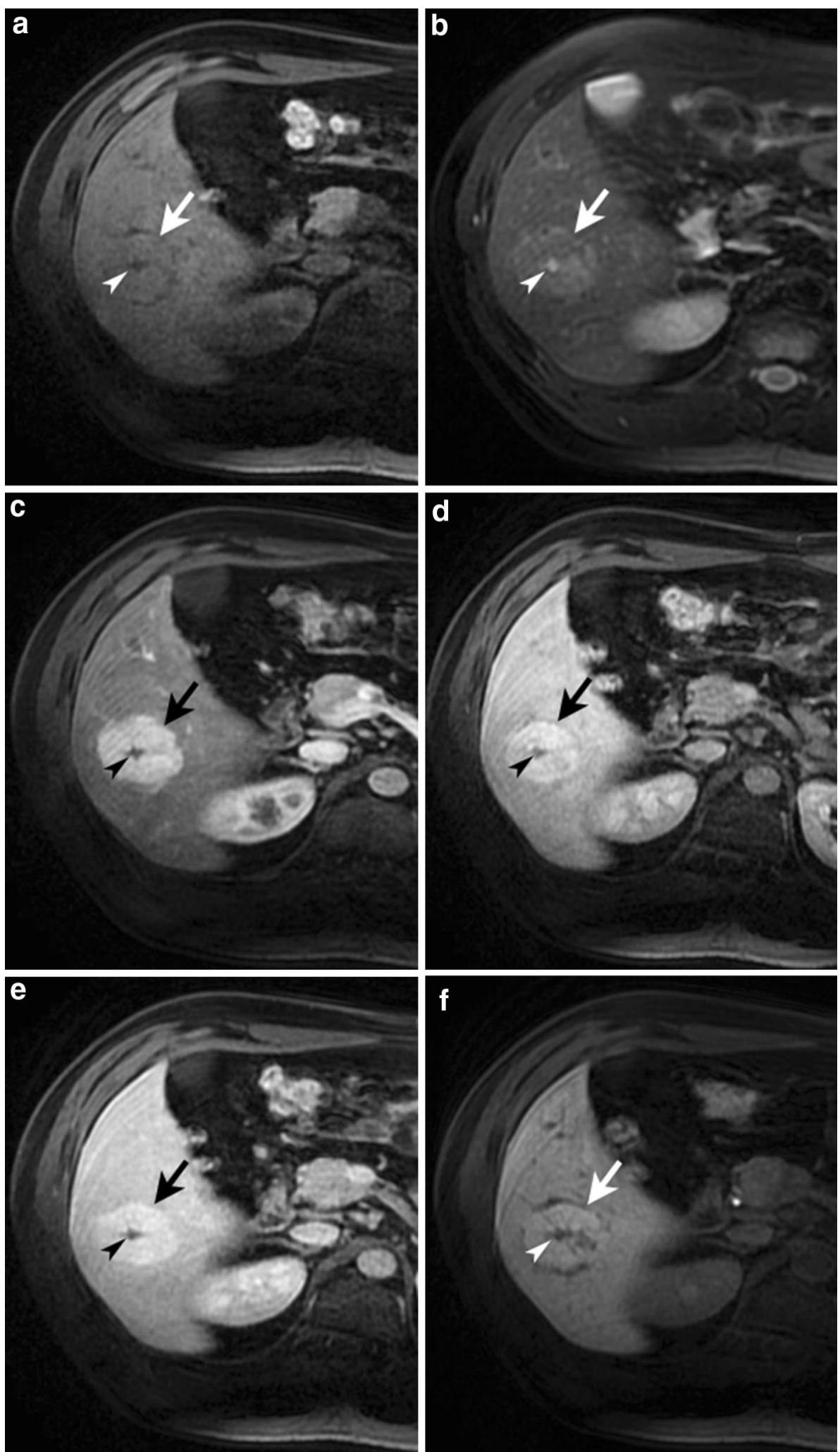

After contrast agent injection, 95/97 (97.9\%) FNHs showed hyperenhancement to varying degrees in comparison with adjacent liver parenchyma in the arterial phase. In the hepatic venous and late phases, all these 95 FNHs were either isoenhancing ( $n=67: 10$ in men and 57 in women) or slightly hyperenhancing ( $n=28$ : seven in men and 21 in women) in comparison with surrounding liver parenchyma (Fig. 3). One FNH appeared isoenhancing 
Table 4 MRI pattern of central scar detected in 20 FNHs

\begin{tabular}{llllllll}
\hline & No. of scars & T1-w & T2-w & Arterial & Hepatic venous & Delayed & Hepato-specific \\
\hline Men & $4 / 18(22.2 \%)$ & 2 Hypo & 2 Hyper & 4 Hypo & 4 Hypo & 1 Hypo & 1 Hypo \\
& & & & & & 1 Hyper \\
Women & $16 / 63(25.4 \%)$ & 9 Hypo & 11 Hyper & 16 Hypo & 2 Hypo & 2 Hypo & 9 Hypo \\
& & & & & 8 Hyper & 7 Hyper \\
$p$ value (scar presence) & & 0.822 & 0.482 & 1.000 & 0.143 & 0.822 & 0.264 \\
$p$ value (scar appearance) & & 1.000 & 1.000 & 1.000 & 0.015 & 0.620 & 1.000 \\
\hline
\end{tabular}

Fig. 3 FNH in a 27-year-old man, contrast-enhanced ultrasound (CEUS). a Baseline US shows a $2.5-\mathrm{cm}$ isoechoic lesion located in the third segment in subcapsular region (arrow). b In the early arterial phase, CEUS depicts the spokewheel sign (arrow). c In the late arterial phase, the lesion shows clear-cut and homogeneous contrast enhancement (arrow). d The lesion appears slightly hyperechoic to the surrounding liver parenchyma in the extended portal venous phase
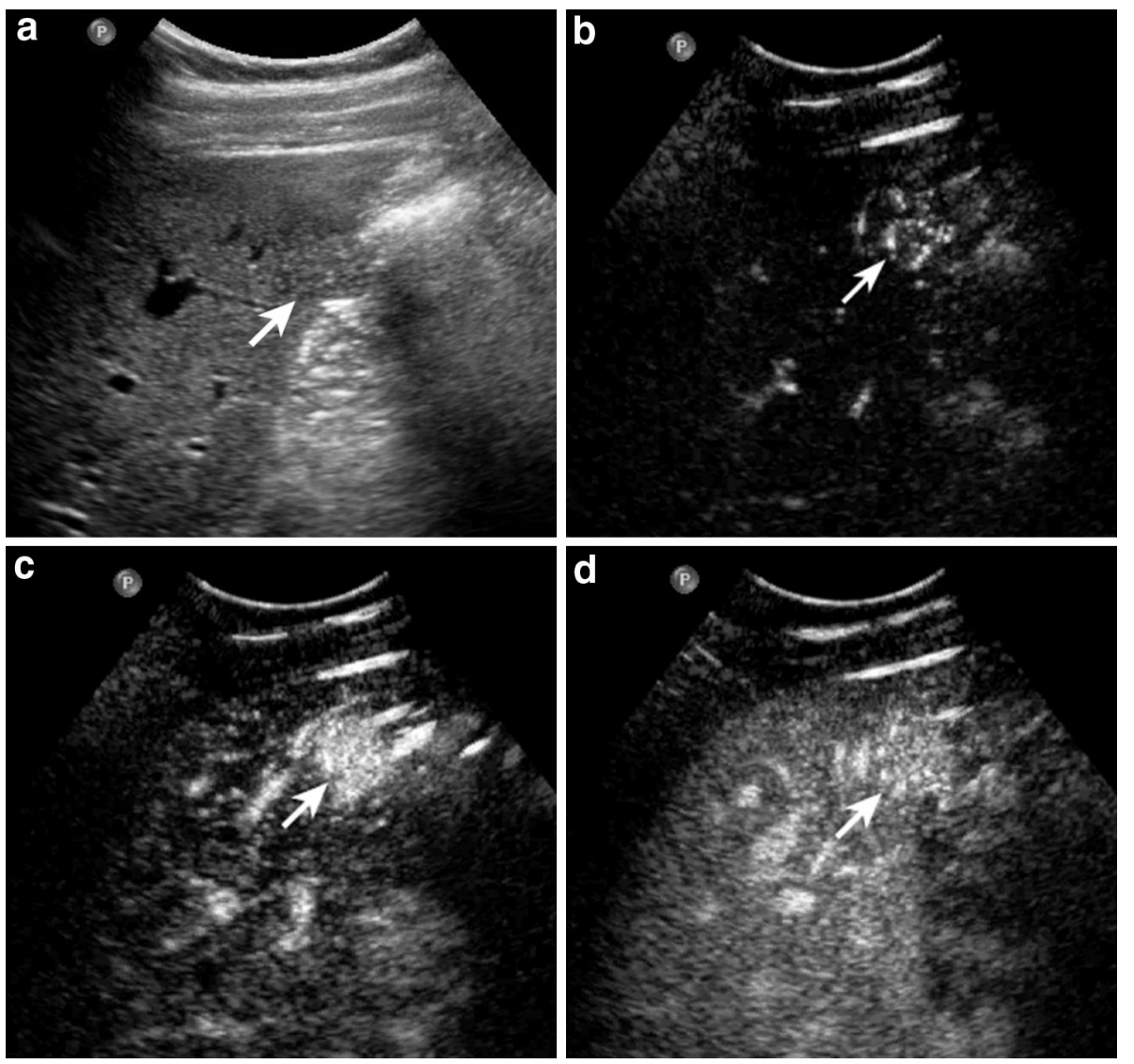

throughout the vascular phase and one FNH (sized $3.6 \mathrm{~cm}$ and located in the subcapsular region of segment VII in a "bright" echogenic liver) remained hypoenhanced throughout the vascular phase, but showed a spoke-wheel sign in the arterial phase.

No statistically significant differences were noted between females and males in contrast-enhancement pattern at CEUS $(p>0.05)$.

At least one sign—among spoke-wheel, central scar, and/or feeding vessel-could be detected at CEUS in 27 out of $97(27.8 \%)$ FNHs. There was no statistically significant difference between men and women: 5/17 (29.4\%) signs in men and 22/80 (27.5\%) signs in women $(p>0.05)$.

\section{Discussion}

In the present study, FNHs occurring in men accounted for $19.8 \%$ of all patients, confirming the higher prevalence in females $(4: 1)$ and the previously reported trend of a higher mean age at diagnosis in men (41 years) in comparison to women (38 years), although this latter finding did not show any statistically significant difference $[1,3]$. Luciani et al. [3] have also reported that FNHs arising in men are smaller than those found in women $(37.5 \mathrm{vs}$. $63.4 \mathrm{~mm}$ on average, respectively) as well as a trend of fewer lesions per patient in men (1.0 vs. 1.3 per patient). Although our series confirm the trend of a smaller size of FNHs in men $(2.7 \mathrm{~cm})$ than in women $(3.1 \mathrm{~cm})$, no statistically significant 
differences were found between the two groups in terms of dimension, as well as in number of lesions per patient (1.22 vs. 1.21 FNH per patient in men and women, respectively) $(p>0.05)$. An explanation for these different results might be related to the monocentric nature of both studies. Further multicentric studies sampling a larger population may be warranted to elucidate this issue.

In our series, no statistically significant differences between men and women were found on unenhanced images from US, MDCT and MRI.

After contrast agent injection, our findings confirm the previously reported strong arterial enhancement on dynamic contrast-enhanced images followed by sustained enhancement, without any statistically significant difference among imaging modalities [5, 7, 10, 13]. In this regard, although the study of Luciani et al. [3] mainly focused on MRI findings, FNHs were reported to be less often typical in men than in women (61.1 vs. $77.8 \%$ ), but the described differences were not statistically significant and no explanation for this finding was provided. Interestingly, in that study the two men with FNH without central stellate area on MR images had small lesions (25 and $30 \mathrm{~mm}$ in diameter, respectively), with no central fibrous area on gross examination of the surgical specimen. Several studies have demonstrated that the detection of typical imaging features of FNH is often strongly, heavily dependent on lesion size, being less frequent in lesions smaller than $3 \mathrm{~cm}$ [5-7]. Consequently, considering our series, it might be hypothesised that the difference found in that study may be, at least in part, more closely related to the different lesion size reported in the two different groups of male and female patients than to real pathological differences. Indeed, the abnormalities found at pathology in men in that study were also described in women [4]. Furthermore, the study by Luciani et al. did not use gadolinium chelate with hepatocellular-specific properties, so the hepatobiliary phase could not be exploited and, eventually, the seven male patients with FNHs lacking at least one typical finding on MRI underwent surgery. It is noteworthy that all the FNHs studied in our series-whether in men or women-showed uptake of hepatocellular-specific contrast agent, demonstrating the hepatocellular nature of the lesions and, in the proper clinical setting, their substantial benignity. Hence, our data also support the hypothesis made by other researchers that exploiting the properties of the newer hepatocellular-specific MR contrast agents enables us to depict the morphological and functional characteristics of FNH noninvasively and may aid in the differential diagnosis of these lesions, thus reducing the need for biopsy and even surgery [13-15].

This retrospective study has some important limitations. First, we had a selection bias. The patients were selected on the basis of their presumed diagnosis in an imaging database. Consequently, those with FNH lesions lacking the characteristic imaging findings may have been missed. Nevertheless, if atypical appearance of FNH is a feature mainly observed in men we should have observed a dramatic reduction of typical FNHs found in men in comparison with women, but this was not the case. Second, the final diagnosis was established in the majority of cases without pathological evaluation because of ethical concerns. However, all lesions were well characterised at CEUS, multiphase contrast-enhanced CT and/or MRI on the basis of the typical enhancement patterns, which we considered to be established diagnostic criteria, as done in other studies [14]. The third limitation was the lack of multi-observer evaluation for imaging analysis.

\section{Conclusions}

Despite the above-mentioned limitations, our results do not show any differences in the imaging features, age of occurrence and size of FNH between men and women.

Conflict of interest Tommaso Vincenzo Bartolotta, Adele Taibbi, Giuseppe Brancatelli, Domenica Matranga, Marisa Tumbarello, Massimo Midiri, Roberto Lagalla declare that they have no conflict of interest related to the publication of this article.

\section{References}

1. Vilgrain V (2006) Focal nodular hyperplasia. Eur J Radiol 58:236-245

2. Ros PR, Menu Y, Vilgrain V et al (2001) Liver neoplasms and tumor-like conditions. Eur Radiol 11(Suppl 2):S145-S165

3. Luciani A, Kobeiter H, Maison P et al (2002) Focal nodular hyperplasia of the liver in men: is presentation the same in men and women? Gut 50:877-880

4. Nguyen BN, Fléjou JF, Terris B et al (1999) Focal nodular hyperplasia of the liver: a comprehensive pathologic study of 305 lesions and recognition of new histologic forms. Am J Surg Pathol 23:1441-1454

5. Bartolotta TV, Taibbi A, Matranga D et al (2010) Hepatic focal nodular hyperplasia: contrast-enhanced ultrasound findings with emphasis on lesion size, depth and liver echogenicity. Eur Radiol 20:2248-2256

6. Hussain SH, Terkivatan T, Zondervan PE et al (2004) Focal nodular hyperplasia: findings at state-of-the-art MR imaging, US, CT, and pathologic analysis. Radiographics 24:3-19

7. Brancatelli G, Federle MP, Grazioli L et al (2001) Focal nodular hyperplasia: CT findings with emphasis on multiphasic helical CT in 78 patients. Radiology 219:61-68

8. World Medical Association (2000) Declaration of Helsinki: ethical principles for medical research involving human subjects. JAMA 284:3043-3045

9. Bartolotta TV, Taibbi A, Midiri M et al (2009) Focal liver lesions: contrast-enhanced ultrasound. Abdom Imaging 34:193-209

10. Kim TK, Jang HJ, Burns PN et al (2008) Focal nodular hyperplasia and hepatic adenoma: differentiation with low-mechanicalindex contrast-enhanced sonography. AJR Am J Roentgenol 190:58-66 
11. Ungermann L, Eliás P, Zizka J et al (2007) Focal nodular hyperplasia: spoke-wheel arterial pattern and other signs on dynamic contrast-enhanced ultrasonography. Eur J Radiol 63:290-294

12. Grazioli L, Morana G, Kirchin MA et al (2005) Accurate differentiation of focal nodular hyperplasia from hepatic adenoma at gadobenate dimeglumine-enhanced MR imaging: prospective study. Radiology 236:166-177

13. Grazioli L, Bondioni MP, Haradome H et al (2012) Hepatocellular adenoma and focal nodular hyperplasia: value of gadoxetic acid-enhanced MR imaging in differential diagnosis. Radiology 262:520-529

14. Purysko AS, Remer EM, Coppa CP et al (2012) Characteristics and distinguishing features of hepatocellular adenoma and focal nodular hyperplasia on gadoxetate disodium-enhanced MRI. AJR Am J Roentgenol 198:115-123

15. Zech CJ, Grazioli L, Breuer J et al (2008) Diagnostic performance and description of morphological features of focal nodular hyperplasia in Gd-EOB-DTPA-enhanced liver magnetic resonance imaging: results of a multicenter trial. Invest Radiol 43:504-511 